\title{
MODULATION-MODE ASSIGNMENT IN SVD-ASSISTED MULTIUSER MIMO-OFDM SYSTEMS
}

\author{
Sebastian Aust ${ }^{1}$, Andreas Ahrens ${ }^{1}$ and César Benavente-Peces ${ }^{2}$ \\ ${ }^{1}$ Hochschule Wismar, University of Technology, Business and Design, Philipp-Müller-Straße 14, 23966 Wismar, Germany \\ ${ }^{2}$ Universidad Politécnica de Madrid, Ctra. Valencia. km. 7, 28031 Madrid, Spain \\ andreas.ahrens@hs-wismar.de,cesar.benavente@upm.es
}

Keywords: Multiple-Input Multiple-Output System, Orthogonal Frequency Division Multiplexing, Singular-Value Decomposition, Bit Allocation, Power Allocation, Wireless Transmission.

Abstract: In order to comply with the demand on increasing available data rates in particular in wireless technologies,
systems with multiple transmit and receive antennas, also called MIMO (multiple-input multiple-output) sys-
tems, have become indispensable for future generations of wireless systems. Due to the strongly increasing
demand in high-data rate transmission systems, frequency non-selective MIMO links have reached a state of
maturity and frequency selective MIMO links are in the focus of interest. In this field, the combination of
MIMO transmission and OFDM (orthogonal frequency division multiplexing) can be considered as an es-
sential part of fulfilling the requirements of future generations of wireless systems. However, single-user
scenarios have reached a state of maturity. By contrast multiple users' scenarios require substantial further
research, where in comparison to ZF (zero-forcing) multiuser transmission techniques, the individual user's
channel characteristics are taken into consideration in this contribution. The performed joint optimization of
the number of activated MIMO layers and the number of transmitted bits per subcarrier along with the appro-
priate allocation of the transmit power shows that not necessarily all user-specific MIMO layers per subcarrier
have to be activated in order to minimize the overall BER under the constraint of a given fixed data throughput.

\section{INTRODUCTION}

The requirements for transmission capacity for speech, data and multimedia information will probably increase continuously in the future. With the limitation of available resources such as transmit power or bandwidth, the demand to increase the spectral efficiency of future transmission systems is clearly recognizable.

Multicarrier transmission such as OFDM (orthogonal frequency division multiplexing) represents a suitable method for the digital signal transmission over linear distorting channels (Bingham, 2000), (van Nee and Prasad, 2000), (Hwang et al., 2003). The available transmission bandwidth is decomposed into a number of narrowband channels in which data streams are transmitted with reduced speed. Furthermore, it offers a great spectral efficiency which is a requirement in current communication systems to transmit at high data rates over band limited channels, specially those aimed at transmitting multimedia content.

However, in order to comply with the demand on increasing available data rates in particular in wire- less technologies, systems with multiple transmit and receive antennas, also called MIMO (multiple-input multiple-output) systems, have become indispensable and can be considered as an essential part of increasing both the achievable capacity and integrity of future generations of wireless systems (Zheng and Tse, 2003), (Kühn, 2006).

Since the capacity of MIMO systems increases linearly with the minimum number of antennas at both, the transmitter as well as the receiver side, MIMO schemes have attracted substantial attention (McKay and Collings, 2005), (Mueller-Weinfurtner, 2002).

With the increasing desire for high-data rate communication, frequency non-selective MIMO links have reached a state of maturity. By contrast, frequency selective MIMO links require substantial further research. Spatial-temporal vector coding (STVC) introduced by RALEIGH seems to be an appropriate candidate for broadband transmission channels. Unfortunately, such solutions appear to be highly complex (Raleigh and Cioffi, 1998), (Raleigh and Jones, 1999). Therefore, complexity-reduced solutions are 
of great interest, where multicarrier transmission such as OFDM combined with multiple transmit and receive antennas seems to be a promising solution to reduce the complexity significantly.

The combination of MIMO transmission and OFDM, as investigated in this work, can be considered as an essential part of fulfilling the requirements of future generations of wireless systems. However single-user scenarios have reached a state of maturity. By contrast multiple users' scenarios in frequencyselective channel conditions require substantial further research (Ahrens and Benavente-Peces, 2010), (Liu et al., 2008).

Considering the entirety of the antennas of all mobile terminals at one end and the antennas of the base station at the other end of the communication link, state of the art interference cancellation is based on a central signal processing unit, e.g. a central unit at the base station, where joint detection can be applied in the uplink (UL) and joint transmission in the downlink (DL), respectively (Meurer et al., 2000), (Choi and Murch, 2004), (Joham et al., 2005). Widely used linear preprocessing techniques such as Minimum Mean Square Error or Zero Forcing (ZF) have attracted a lot of research and have reached a state of maturity, too (Choi and Murch, 2003).

Multiple users' scenarios in frequency-selective MIMO channels using STVC were investigated in (Ahrens and Benavente-Peces, 2010), where, based on the proposed signal processing, multiuser as well as multi-antenna interferences are perfectly eliminated. Therein, it was shown that not necessarily all user-specific MIMO layers have to be activated in order to minimize the overall BER. Furthermore, power allocation was found to be beneficial for minimizing the overall BER.

Against this background, in this paper a SVDassisted multiuser MIMO-ODFM scheme is investigated, where multiuser interferences as well as multiantenna interferences are perfectly eliminated on each subcarrier. Instead of treating all users' channels jointly as in ZF (zero-forcing) multiuser transmission techniques, the investigated solutions take the individual users' channel characteristics into account (Liu et al., 2008). The novel contribution of this paper is that we demonstrate the benefits of amalgamating a suitable choice of activated MIMO layers and number of bits per subcarrier along with the appropriate allocation of the transmit power under the constraint of a given fixed data throughput.

The remaining part of this paper is organized as follows: Section 2 introduces the subcarrier-specific MIMO-OFDM system model, while the proposed solutions of bit and power allocation are discussed in section 3 . The associated performance results are presented and interpreted in section 4. Finally, section 5 provides some concluding remarks.

\section{MIMO-OFDM SYSTEM MODEL}

In this section a subcarrier-specific MIMO-OFDM system model for both single-user and multi-user scenarios is developed where the arising subcarrierspecific interferences are perfectly eliminated. The system model considered in this work consists of a single base station (BS) supporting $K$ mobile stations (MSs). The BS is equipped with $n_{\mathrm{T}}$ transmit antennas, while the $k$ th (with $k=1, \ldots, K$ ) MS has $n_{\mathrm{R} k}$ receive antennas, i.e. the total number of receive antennas including all $K \mathrm{MSs}$ is given by $n_{\mathrm{R}}=\sum_{k=1}^{K} n_{\mathrm{R} k}$.

In order to combat the effects of the frequency selective MIMO channel, OFDM is used as transmission technique (Bahai and Saltzberg, 1999), (Bingham, 2000). Together with a sufficient guard interval length, interferences between the subcarriers can be avoided and only symbols that are transmitted over the different antennas at same subcarrier can interfere each other. Thus, the arising multi-antenna and multiuser interferences between the different data streams, transmitted over the same subcarrier, require appropriate subcarrier-specific signal processing strategies.

\subsection{Single-user System}

Considering a single-user MIMO link $(K=1)$ composed of $n_{\mathrm{T}}$ transmit and $n_{\mathrm{R}}$ receive antennas, the obtained $\left(n_{\mathrm{R}}, n_{\mathrm{T}}\right)$-MIMO-OFDM system transmits an $N$-point IFFT ( $N$ subchannels) modulated data signal over every transmit antenna. The subcarrier-specific system is modelled by

$$
\mathbf{u}^{(\kappa)}=\mathbf{H}^{(\kappa)} \cdot \mathbf{c}^{(\kappa)}+\mathbf{n}^{(\kappa)} .
$$

In (1), the $\left(n_{\mathrm{T}} \times 1\right)$ vector $\mathbf{c}^{(\mathrm{\kappa})}$ contains the complex input symbols transmitted over the $\kappa$ th subcarrier on each input. Applying OFDM with a sufficient guard interval length, the $\left(n_{\mathrm{R}} \times n_{\mathrm{T}}\right)$ matrix $\mathbf{H}^{(\kappa)}$ in (1) results in

$$
\mathbf{H}^{(\kappa)}=\left[\begin{array}{ccc}
h_{11}^{(\kappa)} & \cdots & h_{1 n_{\mathrm{T}}}^{(\kappa)} \\
\vdots & \ddots & \vdots \\
h_{n_{\mathrm{R}} 1}^{(\kappa)} & \cdots & h_{n_{\mathrm{R}} n_{\mathrm{T}}}^{(\kappa)}
\end{array}\right],
$$

with the elements $h_{v \mu}^{(\kappa)}$ describing the couplings of the data symbols on the subchannel $\kappa$. The elements can be ascertained calculating the FFT of the channel impulse response from transmitter $\mu$ to receiver 


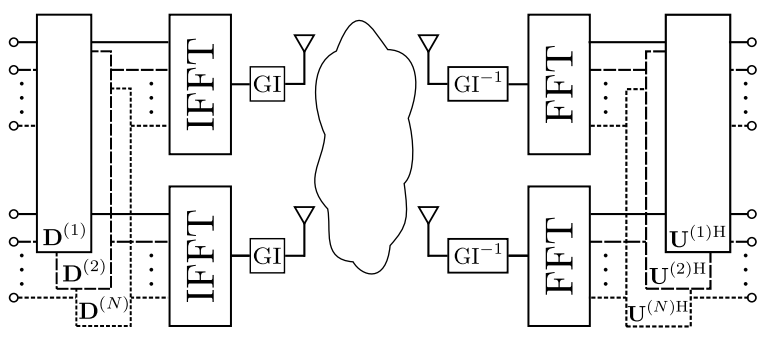

Figure 1: Resulting single-user SVD-based MIMO-OFDM system model with guard interval (GI).

$v$. Finally, $\mathbf{u}^{(\kappa)}$ describes the $\left(n_{\mathrm{R}} \times 1\right)$ received vector and $\mathbf{n}^{(\kappa)}$ is the $\left(n_{\mathrm{R}} \times 1\right)$ vector of the Additive, White Gaussian Noise (AWGN) having a variance of $U_{\mathrm{R}}^{2}$ for both the real and imaginary parts.

The subcarrier-specific interference, introduced by the non-diagonal matrix $\mathbf{H}^{(\kappa)}$, requires appropriate signal processing strategies. A popular technique is based on the singular-value decomposition (SVD) of the matrix $\mathbf{H}^{(\kappa)}$, which can be written as

$$
\mathbf{H}^{(\kappa)}=\mathbf{U}^{(\kappa)} \cdot \mathbf{V}^{(\kappa)} \cdot \mathbf{D}^{(\kappa) \mathrm{H}},
$$

where $\mathbf{U}^{(\kappa)}$ and $\mathbf{D}^{(\kappa)} \mathrm{H}$ are unitary matrices and $\mathbf{V}^{(\kappa)}$ is a real-valued diagonal matrix of the positive square roots of the eigenvalues of the matrix $\mathbf{H}^{(\mathrm{\kappa})} \mathrm{H} \mathbf{H}^{(\kappa)}$ sorted in descending order. The transpose and conjugate transpose (Hermitian) of $\mathbf{D}^{(\kappa)}$ are denoted by $\mathbf{D}^{(\kappa) \mathrm{T}}$ and $\mathbf{D}^{(\kappa)} \mathrm{H}$, respectively. Using $\mathbf{D}^{(\kappa)}$ as preprocessing matrix at the transmitter side and $\mathbf{U}^{(\kappa)} \mathrm{H}$ as postprocessing matrix at the receiver side, the overall transmission relationship results in

$$
\begin{aligned}
\mathbf{y}^{(\kappa)} & =\mathbf{U}^{(\kappa) \mathrm{H}}\left(\mathbf{H}^{(\kappa)} \cdot \mathbf{D}^{(\kappa)} \cdot \mathbf{c}^{(\kappa)}+\mathbf{n}^{(\kappa)}\right) \\
& =\mathbf{V}^{(\kappa)} \cdot \mathbf{c}^{(\kappa)}+\mathbf{w}^{(\kappa)} .
\end{aligned}
$$

Thereby, as $\mathbf{D}^{(\kappa)}$ and $\mathbf{U}^{(\kappa) \mathrm{H}}$ are unitary matrices, neither the transmit power nor the noise power is enhanced. The resulting single-user SVD-based MIMO-OFDM system model is represented in Fig. 1. As a consequence of the processing in (4), the subcarrier-specific channel matrix $\mathbf{H}^{(\kappa)}$ is transformed into independent, non-interfering layers having unequal gains. The resulting subcarrier-specific layer-based MIMO-OFDM system model is highlighted in Fig. 2.

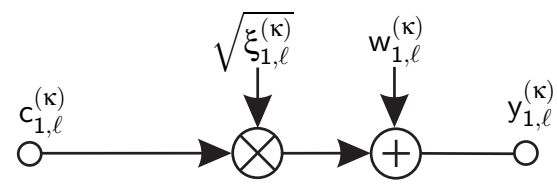

Figure 2: Resulting subcarrier-specific layer-based singleuser MIMO-OFDM system model.
The data symbol $c_{1, \ell}^{(\kappa)}$ to be transmitted over the layer $\ell$ (with $\ell=1,2, \ldots, \min \left(n_{\mathrm{T}}, n_{\mathrm{R}}\right)$ ) at the subcarrier $\kappa($ with $\kappa=1,2, \ldots, N)$ is weighted by the layerspecific factor $\sqrt{\xi_{1, \ell}^{(\kappa)}}$, corresponding to the positive square root of the eigenvalue in $\mathbf{V}^{(\kappa)}$, and together with the additive noise term $w_{1, \ell}^{(\mathrm{\kappa})}$ the received data $y_{1, \ell}^{(\mathrm{\kappa})}$ results in

$$
y_{1, \ell}^{(\kappa)}=\sqrt{\xi_{1, \ell}^{(\kappa)}} \cdot c_{1, \ell}^{(\kappa)}+w_{1, \ell}^{(\kappa)} .
$$

Therein, the number of easily separable layers per subcarrier $L$ is limited by the minimum numbers of antennas at both sides, the transmitter as well as the receiver side, i. e.,

$$
L \leq \min \left(n_{\mathrm{T}}, n_{\mathrm{R}}\right) .
$$

However, it is worth noting that with the aid of powerful non-linear near Maximum Likelihood (ML) sphere decoders it is possible to separate $n_{\mathrm{R}}>n_{\mathrm{T}}$ number of layers (Hanzo and Keller, 2006).

\subsection{Multi-user System}

Now, the subcarrier-specific single-user MIMOOFDM system model $(K=1)$ is extended by considering a single base station (BS) supporting $K$ mobile stations (MSs). The $\left(n_{\mathrm{R} k} \times 1\right)$ user specific symbol vector $\mathbf{c}_{k}^{(\kappa)}$ to be transmitted by the BS over the subcarrier $\mathrm{K}$ is given by

$$
\mathbf{c}_{k}^{(\kappa)}=\left(c_{k, 1}^{(\kappa)}, c_{k, 2}^{(\kappa)}, \ldots, c_{k, n_{\mathrm{R} k}}^{(\kappa)}\right)^{\mathrm{T}} .
$$

The vector $\mathbf{c}_{k}^{(\kappa)}$ is preprocessed before its transmission by multiplying it with the $\left(n_{\mathrm{T}} \times n_{\mathrm{R} k}\right)$ subcarrierspecific DL preprocessing matrix $\mathbf{R}_{k}^{(\kappa)}$ and results in the $\left(n_{\mathrm{T}} \times 1\right)$ user-specific transmit vector

$$
\mathbf{s}_{k}^{(\kappa)}=\mathbf{R}_{k}^{(\kappa)} \mathbf{c}_{k}^{(\kappa)} .
$$

After subcarrier-specific DL transmitter preprocessing, the $n_{\mathrm{T}}$-component signal $\mathbf{s}^{(\mathrm{\kappa})}$ transmitted by the BS specific antennas over the subcarrier $\kappa$ to the $K$ MSs results in

$$
\mathbf{s}^{(\kappa)}=\sum_{k=1}^{K} \mathbf{s}_{k}^{(\kappa)}=\mathbf{R}^{(\kappa)} \mathbf{c}^{(\kappa)},
$$

with the $\left(n_{\mathrm{T}} \times n_{\mathrm{R}}\right)$ preprocessing matrix

$$
\mathbf{R}^{(\kappa)}=\left(\mathbf{R}_{1}^{(\kappa)}, \mathbf{R}_{2}^{(\kappa)}, \ldots, \mathbf{R}_{K}^{(\kappa)}\right) .
$$

In (9), the overall $\left(n_{\mathrm{R}} \times 1\right)$ transmitted DL data vector $\mathbf{c}^{(\kappa)}$ combines all $K$ DL transmit vectors $\mathbf{c}_{k}^{(\kappa)}$ (with $k=$ $1,2, \ldots, K)$ and is given by

$$
\mathbf{c}^{(\kappa)}=\left(\mathbf{c}_{1}^{(\kappa) \mathrm{T}}, \mathbf{c}_{2}^{(\kappa) \mathrm{T}} \ldots, \mathbf{c}_{K}^{(\kappa) \mathrm{T}}\right)^{\mathrm{T}} .
$$


At the receiver side, the $\left(n_{\mathrm{R} k} \times 1\right)$ vector $\mathbf{u}_{k}^{(\kappa)}$ of the $k$ th $\mathrm{MS}$ is given by

$$
\mathbf{u}_{k}^{(\kappa)}=\mathbf{H}_{k}^{(\kappa)} \mathbf{s}^{(\kappa)}+\mathbf{n}_{k}^{(\kappa)}=\mathbf{H}_{k}^{(\kappa)} \mathbf{R}^{(\kappa)} \mathbf{c}^{(\kappa)}+\mathbf{n}_{k}^{(\kappa)} .
$$

and can be expressed by

$$
\mathbf{u}_{k}^{(\kappa)}=\mathbf{H}_{k}^{(\kappa)} \mathbf{R}_{k}^{(\kappa)} \mathbf{c}_{k}^{(\kappa)}+\sum_{i=1, i \neq k}^{K} \mathbf{H}_{k}^{(\kappa)} \mathbf{R}_{i}^{(\kappa)} \mathbf{c}_{i}^{(\kappa)}+\mathbf{n}_{k}^{(\kappa)},
$$

where the MSs received signals at the subcarrier $\kappa$ (with $\kappa=1,2, \ldots, N$ ) experience both multi-user and multi-antenna interferences. In (12), the $\left(n_{\mathrm{R} k} \times n_{\mathrm{T}}\right)$ subcarrier-specific channel matrix $\mathbf{H}_{k}^{(\mathrm{\kappa})}$ connects the $n_{\mathrm{T}}$ BS specific transmit antennas with the $n_{\mathrm{R} k}$ receive antennas of the $k$ th MS.

The subcarrier-specific interference, which is introduced by the off-diagonal elements of the channel matrix $\mathbf{H}_{k}^{(\kappa)}$, requires appropriate signal processing strategies. A popular technique is based on the SVD of the system matrix $\mathbf{H}_{k}^{(\kappa)}$. Upon carrying out the SVD of $\mathbf{H}_{k}^{(\kappa)}$ with $n_{\mathrm{T}} \geq n_{\mathrm{R}}$ and assuming that the rank of the matrix $\mathbf{H}_{k}^{(\kappa)}$ equals $n_{\mathrm{R} k}$, i. e., $\operatorname{rank}\left(\mathbf{H}_{k}^{(\kappa)}\right)=n_{\mathrm{R} k}$, we get

$$
\mathbf{H}_{k}^{(\kappa)}=\mathbf{U}_{k}^{(\kappa)} \cdot \mathbf{V}_{k}^{(\kappa)} \cdot \mathbf{D}_{k}^{(\kappa) \mathrm{H}},
$$

with the $\left(n_{\mathrm{R} k} \times n_{\mathrm{R} k}\right)$ unitary matrix $\mathbf{U}_{k}^{(\kappa)}$ and the $\left(n_{\mathrm{T}} \times n_{\mathrm{T}}\right)$ unitary matrix $\mathbf{D}_{k}^{(\kappa) \mathrm{H}}$, respectively. The $\left(n_{\mathrm{R} k} \times n_{\mathrm{T}}\right)$ diagonal matrix $\mathbf{V}_{k}^{(\mathrm{\kappa})}$ can be decomposed into a $\left(n_{\mathrm{R} k} \times n_{\mathrm{R} k}\right)$ matrix $\mathbf{V}_{k \mathrm{u}}^{(\mathrm{\kappa})}$ containing the nonzero square roots of the eigenvalues of $\mathbf{H}_{k}^{(\mathrm{\kappa}) \mathrm{H}} \mathbf{H}_{k}^{(\mathrm{\kappa})}$, i. e.,

$$
\mathbf{V}_{k \mathrm{u}}^{(\kappa)}=\left[\begin{array}{cccc}
\sqrt{\xi_{k, 1}^{(\kappa)}} & 0 & \cdots & 0 \\
0 & \sqrt{\xi_{k, 2}^{(\kappa)}} & \ddots & \vdots \\
\vdots & \ddots & \ddots & \vdots \\
0 & 0 & \cdots & \sqrt{\xi_{k, n_{\mathrm{R} k}^{(\kappa)}}}
\end{array}\right]
$$

and a $\left(n_{\mathrm{R} k} \times\left(n_{\mathrm{T}}-n_{\mathrm{R} k}\right)\right)$ zero-matrix $\mathbf{V}_{k \mathrm{n}}^{(\boldsymbol{\kappa})}$ according to

$$
\mathbf{V}_{k}^{(\kappa)}=\left(\mathbf{V}_{k \mathrm{u}}^{(\kappa)} \mathbf{V}_{k \mathrm{n}}^{(\kappa)}\right)=\left(\mathbf{V}_{k \mathrm{u}}^{(\kappa)} \mathbf{0}\right)
$$

Additionally, the $\left(n_{\mathrm{T}} \times n_{\mathrm{T}}\right)$ unitary matrix $\mathbf{D}_{k}^{(\kappa)}$ can be decomposed into a $\left(n_{\mathrm{T}} \times n_{\mathrm{R} k}\right)$ matrix $\mathbf{D}_{k \mathrm{u}}^{(\kappa)}$ constituted by the eigenvectors corresponding to the non-zero eigenvalues of $\mathbf{H}_{k}^{(\kappa) \mathrm{H}} \mathbf{H}_{k}^{(\kappa)}$ and a $\left(n_{\mathrm{T}} \times\left(n_{\mathrm{T}}-n_{\mathrm{R} k}\right)\right)$ matrix $\mathbf{D}_{k \mathrm{n}}^{(\kappa)}$ constituted by the eigenvectors corresponding to the zero eigenvalues of $\mathbf{H}_{k}^{(\kappa) \mathrm{H}} \mathbf{H}_{k}^{(\kappa)}$. The decom- position of the matrix $\mathbf{D}_{k}^{(\kappa) \mathrm{H}}$ results in

$$
\mathbf{D}_{k}^{(\kappa) \mathrm{H}}=\left(\begin{array}{c}
\mathbf{D}_{k \mathrm{u}}^{(\kappa) \mathrm{H}} \\
\mathbf{D}_{k \mathrm{n}}^{(\kappa) \mathrm{H}}
\end{array}\right) .
$$

Finally, the subcarrier-specific downlink received signal $\mathbf{u}_{k}^{(\mathrm{\kappa})}$ of the $k$ th MS may be expressed as

$$
\mathbf{u}_{k}^{(\kappa)}=\mathbf{U}_{k}^{(\kappa)} \mathbf{V}_{k \mathrm{u}}^{(\kappa)} \mathbf{D}_{k \mathrm{u}}^{(\kappa) \mathrm{H}} \mathbf{R}^{(\kappa)} \mathbf{c}^{(\kappa)}+\mathbf{n}_{k}^{(\kappa)},
$$

with the vector $\mathbf{n}_{k}^{(\kappa)}$ of the Additive, White Gaussian Noise (AWGN). Taking all MSs received DL signals $\mathbf{u}_{k}^{(\kappa)}$ into account, the $\left(n_{\mathrm{R}} \times 1\right)$ receive vector results in

$$
\mathbf{u}^{(\kappa)}=\left(\mathbf{u}_{1}^{(\kappa) \mathrm{T}}, \mathbf{u}_{2}^{(\kappa) \mathrm{T}}, \ldots, \mathbf{u}_{K}^{(\kappa) \mathrm{T}}\right)^{\mathrm{T}} .
$$

The overall DL signal vector $\mathbf{u}^{(\kappa)}$ including the received signals of all $K$ MSs can be expressed by

$$
\mathbf{u}^{(\kappa)}=\mathbf{U}^{(\kappa)} \mathbf{V}_{\mathrm{u}}^{(\kappa)} \mathbf{D}_{\mathrm{u}}^{(\kappa) \mathrm{H}} \mathbf{R}^{(\kappa)} \mathbf{c}^{(\kappa)}+\mathbf{n}^{(\kappa)},
$$

with the overall $\left(n_{\mathrm{R}} \times 1\right)$ noise vector

$$
\mathbf{n}^{(\kappa)}=\left(\mathbf{n}_{1}^{(\kappa) \mathrm{T}}, \mathbf{n}_{2}^{(\kappa) \mathrm{T}}, \ldots, \mathbf{n}_{K}^{(\kappa) \mathrm{T}}\right)^{\mathrm{T}},
$$

the $\left(n_{\mathrm{R}} \times n_{\mathrm{R}}\right)$ block diagonal matrix $\mathbf{U}^{(\kappa)}$

$$
\mathbf{U}^{(\kappa)}=\left[\begin{array}{cccc}
\mathbf{U}_{1}^{(\kappa)} & \mathbf{0} & \cdots & \mathbf{0} \\
\mathbf{0} & \mathbf{U}_{2}^{(\kappa)} & \ddots & \vdots \\
\vdots & \ddots & \ddots & \vdots \\
\mathbf{0} & \mathbf{0} & \cdots & \mathbf{U}_{K}^{(\kappa)}
\end{array}\right]
$$

the $\left(n_{\mathrm{R}} \times n_{\mathrm{R}}\right)$ block diagonal matrix $\mathbf{V}_{\mathrm{u}}^{(\kappa)}$

$$
\mathbf{V}_{\mathrm{u}}^{(\kappa)}=\left[\begin{array}{cccc}
\mathbf{V}_{1 \mathrm{u}}^{(\kappa)} & \mathbf{0} & \cdots & \mathbf{0} \\
\mathbf{0} & \mathbf{V}_{2 \mathrm{u}}^{(\mathrm{\kappa})} & \ddots & \vdots \\
\vdots & \ddots & \ddots & \vdots \\
\mathbf{0} & \mathbf{0} & \cdots & \mathbf{V}_{K \mathrm{u}}^{(\kappa)}
\end{array}\right],
$$

and the $\left(n_{\mathrm{T}} \times n_{\mathrm{R}}\right)$ matrix $\mathbf{D}_{\mathrm{u}}^{(\kappa)}$ which is given by

$$
\mathbf{D}_{\mathrm{u}}^{(\kappa)}=\left(\mathbf{D}_{1 \mathrm{u}}^{(\kappa)}, \mathbf{D}_{2 \mathrm{u}}^{(\kappa)}, \ldots, \mathbf{D}_{K \mathrm{u}}^{(\kappa)}\right) .
$$

In order to suppress the DL multiuser interferences (MUI) at the subcarrier $\kappa$ perfectly, the DL preprocessing matrix $\mathbf{R}^{(\kappa)}$ has to be designed to satisfy the following condition

$$
\mathbf{D}_{\mathrm{u}}^{(\kappa) \mathrm{H}} \mathbf{R}^{(\kappa)}=\mathbf{P}^{(\kappa)},
$$

with the real-valued $\left(n_{\mathrm{R}} \times n_{\mathrm{R}}\right)$ diagonal matrix $\mathbf{P}^{(\kappa)}$ taking the transmit-power constraint into account. In order to satisfy (25), $\mathbf{R}^{(\kappa)}$ can be defined as follows

$$
\mathbf{R}^{(\kappa)}=\mathbf{D}_{\mathrm{u}}^{(\kappa)}\left(\mathbf{D}_{\mathrm{u}}^{(\kappa) \mathrm{H}} \mathbf{D}_{\mathrm{u}}^{(\kappa)}\right)^{-1} \mathbf{P}^{(\kappa)} .
$$


Taking the ZF design criterion for the DL preprocessing matrix into account, the matrix $\mathbf{P}^{(\mathrm{\kappa})}$ simplifies to an $\left(n_{\mathrm{R}} \times n_{\mathrm{R}}\right)$ diagonal matrix, i.e. $\mathbf{P}^{(\kappa)}=$ $\sqrt{\beta^{(\kappa)}} \mathbf{I}_{n_{\mathrm{R}} \times n_{\mathrm{R}}}$, with the parameter $\sqrt{\beta^{(\kappa)}}$ describing the transmit-power constraint. When taking the DL preprocessing matrix, defined in (26), into account, the overall subcarrier-specific received vector of all $K$ MSs, defined in (20), can be simplified to

$$
\mathbf{u}^{(\kappa)}=\mathbf{U}^{(\kappa)} \mathbf{V}_{\mathrm{u}}^{(\kappa)} \mathbf{P}^{(\kappa)} \mathbf{c}^{(\kappa)}+\mathbf{n}^{(\kappa)} .
$$

Therein, the $\left(n_{\mathrm{R}} \times n_{\mathrm{R}}\right)$ block diagonal matrix $\mathbf{P}^{(\kappa)}$ is given by

$$
\mathbf{P}^{(\kappa)}=\left[\begin{array}{cccc}
\mathbf{P}_{1}^{(\kappa)} & \mathbf{0} & \cdots & \mathbf{0} \\
\mathbf{0} & \mathbf{P}_{2}^{(\kappa)} & \ddots & \vdots \\
\vdots & \ddots & \ddots & \vdots \\
\mathbf{0} & \mathbf{0} & \cdots & \mathbf{P}_{K}^{(\kappa)}
\end{array}\right] .
$$

In (27), the user-specific $\left(n_{\mathrm{R} k} \times 1\right)$ vector $\mathbf{u}_{k}^{(\kappa)}$ can be expressed as

$$
\mathbf{u}_{k}^{(\kappa)}=\mathbf{U}_{k}^{(\kappa)} \mathbf{V}_{k \mathrm{u}}^{(\kappa)} \mathbf{P}_{k}^{(\kappa)} \mathbf{c}_{k}^{(\kappa)}+\mathbf{n}_{k}^{(\kappa)},
$$

with the user-specific $\left(n_{\mathrm{R} k} \times n_{\mathrm{R} k}\right)$ power allocation matrix

$$
\mathbf{P}_{k}^{(\kappa)}=\left[\begin{array}{cccc}
\sqrt{p_{k, 1}^{(\kappa)}} & 0 & \cdots & 0 \\
0 & \sqrt{p_{k, 2}^{(\kappa)}} & \ddots & \vdots \\
\vdots & \ddots & \ddots & \vdots \\
0 & 0 & \cdots & \sqrt{p_{k, n_{\mathrm{R} k}}^{(\kappa)}}
\end{array}\right] .
$$

As long as the transmit power is uniformly distributed over the number of activated MIMO layers per subcarrier, the matrix $\mathbf{P}_{k}^{(\kappa)}$ simplifies to

$$
\mathbf{P}_{k}^{(\kappa)}=\sqrt{\beta^{(\kappa)}} \mathbf{I}_{n_{\mathrm{R} k} \times n_{\mathrm{R} k}} .
$$

After postprocessing of the received signal vectors $\mathbf{u}_{k}^{(\kappa)}$ with the corresponding unitary matrix $\mathbf{U}_{k}^{(\kappa) H}$, the user-specific decision variables at the subcarrier $\kappa$ result with $\mathbf{U}_{k}^{(\kappa) \mathrm{H}} \mathbf{n}_{k}^{(\kappa)}=\mathbf{w}_{k}^{(\kappa)}$ in

$$
\mathbf{y}_{k}^{(\kappa)}=\mathbf{U}_{k}^{(\kappa)} \mathrm{H}_{\mathbf{u}_{k}^{(\kappa)}}=\mathbf{V}_{k \mathrm{u}}^{(\kappa)} \mathbf{P}_{k}^{(\kappa)} \mathbf{c}_{k}^{(\kappa)}+\mathbf{w}_{k}^{(\kappa)},
$$

or alternatively for the whole system with $\mathbf{U}^{(\kappa)} \mathrm{H}^{(\kappa)}=\mathbf{w}^{(\kappa)}$ in

$$
\mathbf{y}^{(\kappa)}=\mathbf{U}^{(\kappa) \mathrm{H}} \mathbf{u}^{(\kappa)}=\mathbf{V}_{\mathrm{u}}^{(\kappa)} \mathbf{P}^{(\kappa)} \mathbf{c}^{(\kappa)}+\mathbf{w}^{(\kappa)},
$$

where subcarrier-specific interferences between the different antenna data streams as well as MUI imposed by the other users are avoided. The resulting multiuser SVD-based MIMO-OFDM system model is represented in Fig. 3, whereas the resulting layerspecific system model is depicted in Fig. 4

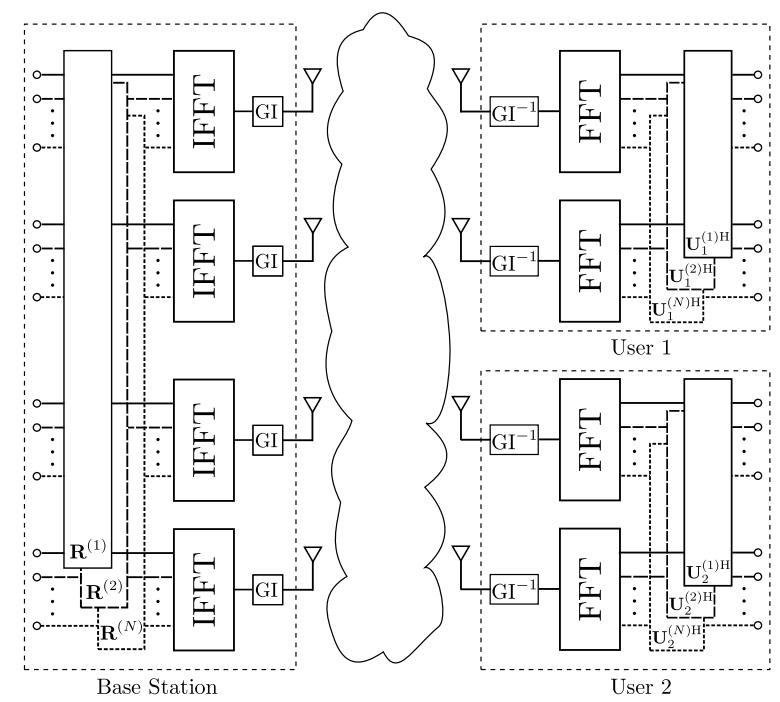

Figure 3: Resulting multiuser SVD-based MIMO-OFDM system model with guard interval (GI).

\section{OPTIMIZATION OBJECTIVES}

In general, the user-specific quality of data transmission can be informally assessed by using the signalto-noise ratio (SNR) at the detector's input defined by the half vertical eye opening and the noise power per quadrature component according to

$$
\rho=\frac{(\text { Half vertical eye opening })^{2}}{\text { Noise Power }}=\frac{\left(U_{\mathrm{A}}\right)^{2}}{\left(U_{\mathrm{R}}\right)^{2}},
$$

which is often used as a quality parameter (Ahrens and Lange, 2008). The relationship between the signal-to-noise ratio $\rho=U_{\mathrm{A}}^{2} / U_{\mathrm{R}}^{2}$ and the bit-error probability evaluated for AWGN channels and $M$-ary Quadrature Amplitude Modulation (QAM) is given by (Proakis, 2000)

$$
P_{\mathrm{BER}}=\frac{2}{\log _{2}(M)}\left(1-\frac{1}{\sqrt{M}}\right) \operatorname{erfc}\left(\sqrt{\frac{\rho}{2}}\right) .
$$

When applying the proposed system structure for the $k$ th user, depicted in Fig. 4, the applied signal processing leads to different eye openings per activated MIMO layer $\ell$ (with $\ell=1,2, \ldots, L$ and $L \leq n_{\mathrm{R} k}$ describing the number of activated user-specific MIMO

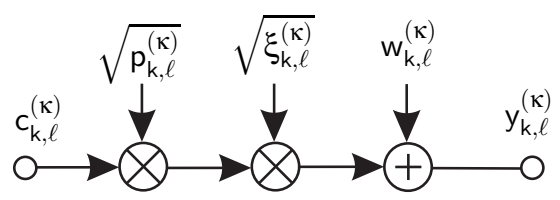

Figure 4: Resulting $k$ th user system model per MIMO layer $\ell$ (with $\ell=1,2, \ldots, n_{\mathrm{R} k}$ ) on subcarrier $\kappa$ (with $\kappa=$ $1,2, \ldots, N)$ 
layers) and per subcarrier $\kappa($ with $\kappa=1,2, \ldots, N)$ according to

$$
U_{\mathrm{A} k \ell}^{(\kappa)}=\sqrt{p_{k, \ell}^{(\kappa)}} \cdot \sqrt{\xi_{k, \ell}^{(\kappa)}} \cdot U_{\mathrm{s} k \ell}^{(\kappa)},
$$

where $U_{\mathrm{s} k \ell}^{(\kappa)}$ denotes the $k$ th user and $\kappa$ th subcarrier specific half-level transmit amplitude assuming $M_{\ell}$-ary QAM, $\sqrt{\xi_{k, \ell}^{(\kappa)}}$ represents the corresponding subcarrier-specific positive square roots of the eigenvalues of the matrix $\mathbf{H}_{k}^{(\mathrm{\kappa}) \mathrm{H}} \mathbf{H}_{k}^{(\mathrm{\kappa})}$ and $\sqrt{p_{k, \ell}^{(\kappa)}}$ represents the corresponding power allocation weighting parameters (Fig. 4). Together with the noise power per quadrature component, introduced by the additive, white Gaussian noise (AWGN) vector $\mathbf{w}_{k}^{(\mathrm{\kappa})}$ in (32), the $k$ th user-specific SNR per MIMO layer $\ell$ and subcarrier $\kappa$ becomes

$$
\rho_{k \ell}^{(\kappa)}=\frac{\left(U_{\mathrm{A} k \ell}^{(\kappa)}\right)^{2}}{U_{\mathrm{R}}^{2}} .
$$

Realizing a parallel transmission over $L$ MIMO layers and taking all $N$ subcarriers into account, the overall mean user-specific transmit power becomes

$$
P_{\mathrm{s} k}=\sum_{\kappa=1}^{N} \sum_{\ell=1}^{L} P_{\mathrm{s} k \ell}^{(\kappa)} .
$$

Considering QAM constellations, the average userspecific transmit power $P_{\mathrm{s} k \ell}^{(\mathrm{\kappa})}$ per MIMO layer $\ell$ and subcarrier $\kappa$ may be expressed as (Proakis, 2000)

$$
P_{\mathrm{s} k \ell}^{(\kappa)}=\frac{2}{3}\left(U_{\mathrm{s} k \ell}^{(\mathrm{\kappa})}\right)^{2}\left(M_{k \ell}^{(\kappa)}-1\right) .
$$

Combining (37) and (39) together with (36), the layerspecific SNR at the subcarrier $\kappa$ results in

$$
\rho_{k \ell}^{(\kappa)}=p_{k, \ell}^{(\kappa)} \xi_{k, \ell}^{(\kappa)} \frac{3}{2\left(M_{k \ell}^{(\kappa)}-1\right)} \frac{P_{\mathrm{s} k \ell}^{(\kappa)}}{U_{\mathrm{R}}^{2}} .
$$

Assuming that the user-specific transmit power is uniformly distributed over the number of activated MIMO layers and subcarriers, the quality on each subcarrier is affected by both, the choice of the QAMconstellation sizes per layer and the layer-specific weighting factors. In order to transmit at a fixed data rate while maintaining the best possible integrity, i. e., bit-error rate, an appropriate number of user-specific MIMO layers has to be used, which depends on the specific transmission mode, as detailed in Table 1 for the exemplarily investigated two-user MIMO-OFDM system $\left(n_{\mathrm{R} k}=4(\right.$ with $\left.k=1,2), K=2, n_{\mathrm{R}}=n_{\mathrm{T}}=8\right)$.

An optimized adaptive scheme would now use the particular transmission modes on each subcarrier,
Table 1: Investigated user-specific transmission modes per subcarrier.

\begin{tabular}{ccccc}
\hline throughput & layer 1 & layer 2 & layer 3 & layer 4 \\
\hline $8 \mathrm{bit} / \mathrm{s} / \mathrm{Hz}$ & 256 & 0 & 0 & 0 \\
$8 \mathrm{bit} / \mathrm{s} / \mathrm{Hz}$ & 64 & 4 & 0 & 0 \\
$\mathbf{8} \mathrm{bit} / \mathrm{s} / \mathrm{Hz}$ & $\mathbf{1 6}$ & $\mathbf{1 6}$ & $\mathbf{0}$ & $\mathbf{0}$ \\
$\mathbf{8} \mathrm{bit} / \mathrm{s} / \mathrm{Hz}$ & $\mathbf{1 6}$ & $\mathbf{4}$ & $\mathbf{4}$ & $\mathbf{0}$ \\
$8 \mathrm{bit} / \mathrm{s} / \mathrm{Hz}$ & 4 & 4 & 4 & 4 \\
\hline
\end{tabular}

e.g., by using bit auction procedures (Wong et al., 1999), that results in the lowest BER for each MIMOOFDM data vector. However, this would lead to a high signaling overhead. Therefore, in order to avoid any signalling overhead, fixed transmission modes are used in this contribution regardless of the channel quality.

However, it is worth mentioning that in systems where channel state information is available at the transmitter side, the knowledge about how the symbols are attenuated by the channel can be used to adapt the transmit parameters. In this context, power allocation (PA) can be used to balance the bit-error probabilities in the number of activated MIMO layers.

Applying PA, the information about how the symbols are attenuated by the channel, i. e., the singularvalues, has to be sent via a feedback channel to the transmitter side and leads to a signalling overhead that is contradictory to the fix transmission modes that require no signalling overhead. However, as shown in (Ahrens and Lange, 2009) a vector quantizer (VQ) can be used to keep the signalling overhead moderate. Here, a VQ for the power allocation parameters instead of the singular values guarantees a better adaption at a given codebook size, since the power level vectors has less or equal dimensions than the singularvalue vectors (Ahrens and Lange, 2009). Moreover, its elements are much smaller digits ranged from 0 to 1 , rather than from 0 to $+\infty$ in the singular-value vector case. Hence, the entropy of the power level vectors is smaller, which benefits the quantization accuracy and the feedback overhead.

Therefore, besides the choice of the transmission modes, PA can be used to adjust the bit-error probabilities in the activated user-specific MIMO layers and has been widely investigated in the literature (Krongold et al., 2000), (Ahrens and Lange, 2008), (Jang and Lee, 2003).

Analysing the considered MIMO-OFDM system, the user-specific BER of the MIMO-OFDM system is dominated by the specific layers having the lowest SNR's. As a remedy, a MIMO-layer transmit PA scheme is required for minimizing the overall BER 
under the constraint of a limited total MIMO transmit power.

The proposed PA scheme scales the $k$ th user halflevel transmit amplitude $U_{\mathrm{s} k \ell}^{(\mathrm{\kappa})}$ of the $\ell$ th MIMO layer on the subcarrier $\kappa$ by the factor $\sqrt{\tilde{p}_{k, \ell}^{(\kappa)}}$. This results in a MIMO layer-specific transmit amplitude of $U_{\mathrm{s} k \ell}^{(\kappa)} \sqrt{\tilde{p}_{k, \ell}^{(\kappa)}}$ for the QAM symbol of the transmit data vector transmitted at the subcarrier $\kappa$ over the MIMO layer $\ell$. Together with the DL preprocessing design, the layer-specific power allocation parameter at the subcarrier $\kappa$ results in:

$$
\sqrt{p_{k, \ell}^{(\kappa)}}=\sqrt{\beta^{(\kappa)}} \sqrt{\tilde{p}_{k, \ell}^{(\kappa)}} .
$$

PA has been widely investigated in the literature, where optimum solutions tends to be highly complex (Ahrens and Lange, 2007). In this work a suboptimum solution is presented which focusses on the userspecific subcarriers.

A natural choice is to opt for a PA scheme, which results in an identical signal-to-noise ratio

$$
\rho_{\mathrm{PA} k \ell}^{(\kappa)}=\frac{\left(U_{\mathrm{PA} k \ell}^{(\kappa)}\right)^{2}}{U_{\mathrm{R}}^{2}}=\tilde{p}_{k, \ell}^{(\kappa)} \frac{3 \xi_{k, \ell}^{(\kappa)} \beta^{(\kappa)}}{L N\left(M_{k \ell}^{(\kappa)}-1\right)} \frac{E_{\mathrm{s}}}{N_{0}}
$$

for all activated user-specific MIMO layers as shown in (Ahrens and Lange, 2007), i. e., in

$$
\rho_{\mathrm{PA} k \ell}^{(\kappa)}=\mathrm{constant}
$$

for $\ell=1,2, \ldots, L$ and $\kappa=1,2, \ldots, N$. In this particular case, nearly the same BER can be achieved on all activated user-specific MIMO layers and subcarriers (Ahrens and Lange, 2007).

The proposed suboptimum PA scheme focusses on the arguments of the complementary error function and neglects the transmission mode specific factors in front of the complementary error function. However, their influence, introduced by the layer-specific QAM constellation sizes, is by far too small to generate remarkable differences in the performance as shown in (Ahrens and Lange, 2007).

\section{RESULTS}

In this contribution the efficiency of fixed userspecific transmission modes on each subcarrier is studied regardless of the channel quality. Assuming predefined transmission modes, a fixed data rate can be guaranteed. In order to obtain numerical results for the analyzed subcarrier-specific signal processing strategies, a two-path channel model is investigated under time-variant conditions between any

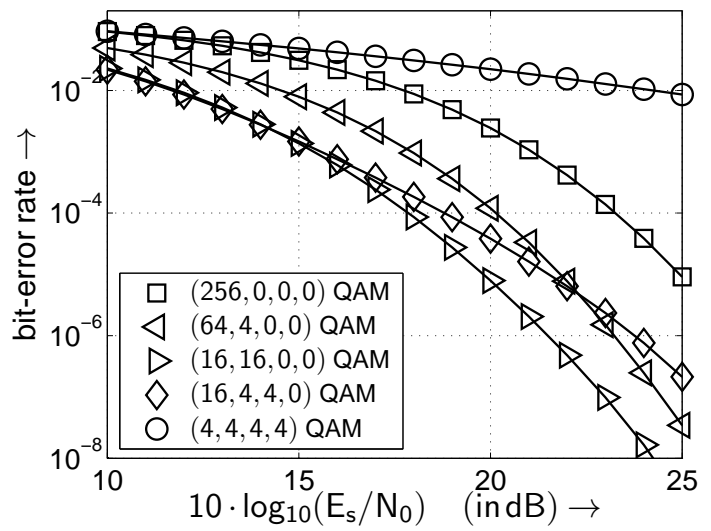

Figure 5: BER without PA when using the transmission modes introduced in Tab. 1 and transmitting $8 \mathrm{bit} / \mathrm{s} / \mathrm{Hz}$ per subcarrier.

given transmit and receive antenna combination. The exemplary impulse response between the $\mu$ th transmit and $v$ th receive antenna as a function of the multicarrier symbol duration $T_{\mathrm{s}}$ is given by

$$
g_{\mathrm{k}}^{(v \mu)}(t, \tau)=g_{\mathrm{k}, 0}(t) \cdot \delta(\tau)+g_{\mathrm{k}, 1}(t) \cdot \delta\left(\tau-3 / 2 T_{\mathrm{s}}\right)
$$

whereby the time-variant behaviour is described by $g_{\mathrm{k}, 0}(t)$ and $g_{\mathrm{k}, 1}(t)$, respectively. It is assumed that the path amplitudes have the same averaged power and undergo a Rayleigh distribution (Pätzold, 2002). Furthermore, a block fading channel model is applied, i. e., the channel is assumed to be time invariant for the duration of one MIMO-OFDM data vector including a guard interval length of $T_{\mathrm{g}}=T_{\mathrm{s}} / 2$. The number of subcarrier of the investigated OFDM system was exemplarily chosen to be $N=4$.

\subsection{Single-user System}

Considering a single-user MIMO-OFDM link $(K=1)$ composed of $n_{\mathrm{T}}=4$ transmit and $n_{\mathrm{R}}=4$ receive antennas, the corresponding calculated BER curves are depicted in Fig. 5 for the different subcarrier-specific QAM constellation sizes and MIMO configurations of Table 1, when transmitting at a fixed bandwidth efficiency of $8 \mathrm{bit} / \mathrm{s} / \mathrm{Hz}$ per subcarrier.

Assuming a uniform distribution of the transmit power over the number of activated MIMO layers per subcarrier, it still turns out that not all subcarrierspecific MIMO layers have to be activated in order to achieve the best system performance.

However, the lowest BERs can only be achieved by using bit auction procedures leading to a high signalling overhead (Wong et al., 1999). Analyzing the probability of choosing subcarrier-specific transmission modes by using optimal bitloading, as depicted 
in Table 2, it turns out that only an appropriate number of subcarrier-specific MIMO layers has to be activated to reach the best results, e.g., the $(16,4,4,0)$ QAM configuration. Therefore, the results, obtained by using bit auction procedures, justify the choice of fixed subcarrier-specific transmission modes regardless of the channel quality as investigated in this contribution.

Table 2: Probability of choosing subcarrier-specific transmission modes at a fixed data rate by using optimal bitloading $\left(10 \cdot \lg \left(E_{\mathrm{S}} / N_{0}\right)=10 \mathrm{~dB}\right.$ and $\left.\kappa=3\right)$.

\begin{tabular}{ccccc}
\hline mode & $(16,4,4,0)$ & $(16,16,0,0)$ & $(64,4,0,0)$ & $(4,4,4,4)$ \\
\hline pdf & 0.722 & 0.261 & 0.016 & 0.000 \\
\hline
\end{tabular}

Further improvements can be achieved by taking PA into account. Adaptive power allocation has been widely investigated in the literature (Krongold et al., 2000), (Jang and Lee, 2003), (Park and Lee, 2004), (Ahrens and Lange, 2008) and can be used to balance the bit-error probabilities in the different number of activated user-specific MIMO layers. Using power allocation, the resulting subcarrier-specific layer-based single-user MIMO-OFDM system model is depicted in Fig. 6.

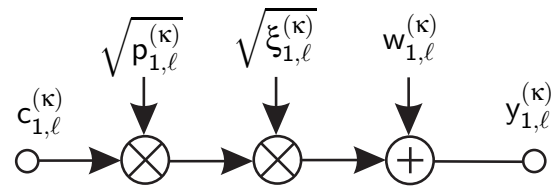

Figure 6: Resulting subcarrier-specific layer-based singleuser MIMO-OFDM system model using Power Allocation.

A common strategy is to use the Lagrange multiplier method in order to find the optimal value of $\sqrt{p_{1, \ell}^{(\kappa)}}$ for each MIMO layer $\ell$ and subcarrier $\kappa$, which often leads to excessive-complexity optimization problems (Ahrens and Lange, 2007). Therefore, suboptimal power allocation strategies having a lower complexity are of common interest (Ahrens and Lange, 2007), (Park and Lee, 2004).

A natural choice is to opt for a PA scheme, which results in an identical signal-to-noise ratio

$$
\rho_{\mathrm{PA} 1 \ell}^{(\kappa)}=\frac{\left(U_{\mathrm{PA} 1 \ell}^{(\kappa)}\right)^{2}}{U_{\mathrm{R}}^{2}}=\tilde{p}_{1, \ell}^{(\kappa)} \frac{3 \xi_{1, \ell}^{(\kappa)}}{L N\left(M_{1 \ell}^{(\kappa)}-1\right)} \frac{E_{\mathrm{s}}}{N_{0}}
$$

for all activated MIMO layers $\ell$ and subcarriers $\kappa$, i. e., in

$$
\rho_{\mathrm{PA} 1 \ell}^{(\kappa)}=\mathrm{constant},
$$

for $\ell=1,2, \ldots, L$ and $\kappa=1,2, \ldots, N$. The power to be allocated to each activated MIMO layer $\ell$ and subarrier $\kappa$ can be shown to be calculated by inverting

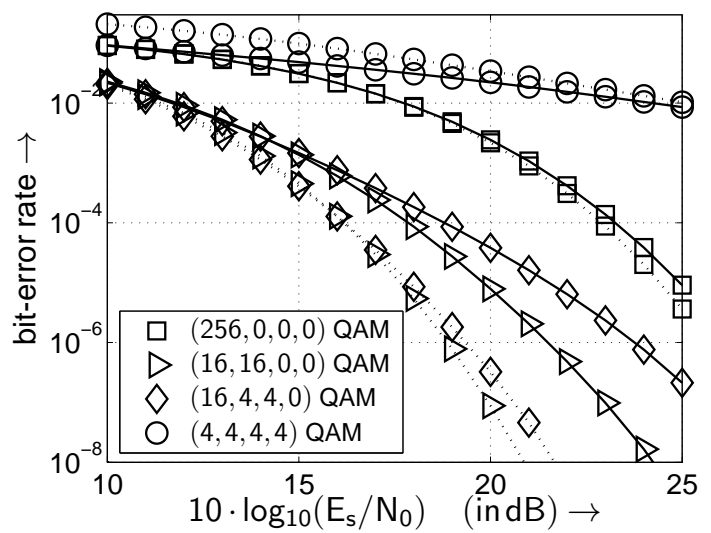

Figure 7: BER with PA (dotted line) and without PA (solid line) when using the transmission modes introduced in Tab. 1 and transmitting 8 bit/s/Hz per subcarrier.

the layer-specific part $\xi_{1, \ell}^{(\kappa)} /\left(M_{1 \ell}^{(\kappa)}-1\right)$ and taking the transmit power constraint into account as presented in (Ahrens and Lange, 2007). In this case for each symbol of the transmitted MIMO-OFDM symbol vector the same half vertical eye opening can be guaranteed, i.e.,

$$
U_{\mathrm{PA} 1 \ell}^{(\mathrm{\kappa})}=\mathrm{constant} .
$$

for all activated MIMO layers $\ell$ (with $\ell=1,2, \ldots, L$ ) and all subcarrier $\kappa$ (with $\kappa=1,2, \ldots, N)$. When assuming an identical detector input noise variance for each channel output symbol, the above-mentioned equal quality scenario (46) is encountered. The only difference between an optimum PA and the here considered equal quality scenario is the consideration of the factor

$$
1-1 / \sqrt{M_{1 \ell}^{(\kappa)}}
$$

in front of the complementary error function by the optimum PA. However as shown by (Ahrens and Lange, 2007) their influence is by far too small to generate remarkable differences in the performance.

Analyzing (46) for a given MIMO-OFDM data block, nearly the same BER can be achieved on all activated MIMO layers and subcarriers. However, taking the time-variant nature of the transmission channel into account, different BERs arise for different MIMO-OFDM data blocks. Therefore, the BER of the MIMO-OFDM system is mainly dominated by the data blocks having the lowest SNRs. In order to overcome this problem, the number of transmit or receive antennas has to be increased, or coding over the different data blocks should be used (Ahrens et al., 2008).

As shown in Fig. 7, taking all subcarrier into account, unequal PA is only effective in conjunction with the optimum number of MIMO layers and at high SNR. 


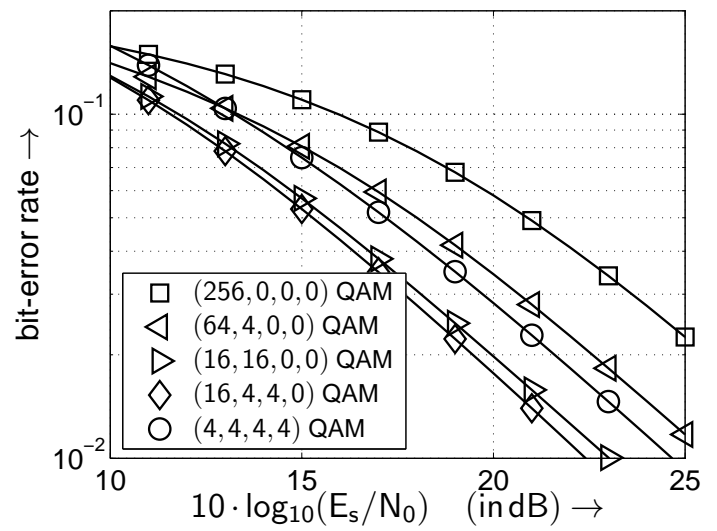

Figure 8: SVD-based user-specific BERs without PA when using the transmission modes introduced in Table 1 and transmitting $8 \mathrm{bit} / \mathrm{s} / \mathrm{Hz}$ per subcarrier.

\subsection{Multi-user System}

The parameters of the analyzed two-users MIMO system are chosen as follows: $P_{\mathrm{s} k}=1 \mathrm{~V}^{2}, n_{\mathrm{R} k}=4$ (with $k=1,2), K=2, n_{\mathrm{R}}=n_{\mathrm{T}}=8$. In this contribution a power with the dimension (voltage) ${ }^{2}$ (in $\mathrm{V}^{2}$ ) is used. At a real constant resistor this value is proportional to the physical power (in W).

The obtained user-specific BER curves are depicted in Fig. 8 for the different subcarrier-specific QAM constellation sizes and MIMO configurations of Table 1 and confirm the obtained results within the single-user MIMO-OFDM system $(K=1)$. Based on the higher total subcarrier-specific throughput within the given bandwidth compared to the single-user system, the gap between the different transmission modes becomes smaller.

Assuming a uniform distribution of the transmit power over the number of activated MIMO layers, it still turns out that not all MIMO layers per subcarrier have to be activated in order to achieve the best BERs. This can still be confirmed by analyzing the probability of choosing user-specific transmission modes within the multiuser DL MIMO-OFDM system by using optimal bitloading (Wong et al., 1999), as depicted in Table 3.

The partitioning of the transmit power to the sub-

Table 3: Probability of choosing user-specific transmission modes $(K=2)$ per subcarrier at a fixed data rate by using optimal bitloading $\left(10 \cdot \log _{10}\left(E_{\mathrm{S}} / N_{0}\right)=10 \mathrm{~dB}\right.$ and $\left.\kappa=3\right)$.

\begin{tabular}{ccccc}
\hline mode & $(16,4,4,0)$ & $(16,16,0,0)$ & $(64,4,0,0)$ & $(4,4,4,4)$ \\
\hline pdf & 0.950 & 0.010 & 0.000 & 0.039 \\
\hline
\end{tabular}

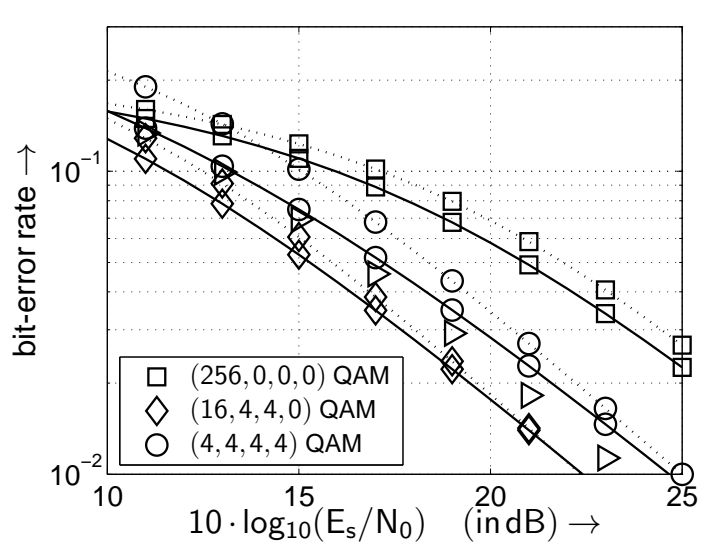

Figure 9: SVD-based user-specific BERs with PA (dotted line) and without PA (solid line) when using the transmission modes introduced in Table 1 and transmitting $8 \mathrm{bit} / \mathrm{s} / \mathrm{Hz}$ per subcarrier.

channels is a degree of freedom in the multiuser system too and has to be done in such a manner, that for the whole system a maximum performance with respect to a given quality criterion and given boundary conditions can be achieved (e.g. minimum biterror rate with limited transmit power). In the proposed multiuser MIMO-OFDM system, user-specific PA can be done, i. e., the available transmit power per user $P_{\mathrm{s} k}=1 \mathrm{~V}^{2}$ can be distributed over all activated user-specific layers on all subcarrier. The obtained BER curves are depicted in Fig. 9.

As developed within the single-user MIMOOFDM system, unequal PA based on the proposed equal-quality scenario is only effective in conjunction with the optimum number of MIMO layers and at high SNR. At the low SNR, as depicted in Fig. 9, the proposed equal-quality scenario doesn't lead to any improvement. Here our results suggest that a uniform distribution of the transmit power seems to be a good choice for minimizing the overall BER.

\section{CONCLUSIONS}

In this paper, the DL performance of a multiuser MIMO-OFDM system is investigated theoretically and by software simulation. Frequency selective MIMO channels are considered and conditions to eliminate multiuser and multi-antenna interferences on each subcarrier are established using the SVD of the individual user subcarrier-specific channel matrix. Furthermore, bit and power allocation in multiuser MIMO-OFDM systems were investigated for constant data throughput per subcarrier. Here, it turned out that the choice of the number of bits per symbol 
as well as the number of activated MIMO layers per subcarrier substantially affects the performance of a MIMO-OFDM system, suggesting that not all userspecific MIMO layers per subcarrier have to be activated in order to achieve the best BERs. Additionally, unequal PA was found to be effective in conjunction with the optimum number of MIMO layers for the overall performance at the high SNR.

\section{REFERENCES}

Ahrens, A. and Benavente-Peces, C. (2010). ModulationMode and Power Assignment for SVD-assisted and Iteratively Detected Downlink Multiuser MIMO Systems. In International Conference on Wireless Information Networks and Systems (WINSYS), pages 107114, Athens (Greece).

Ahrens, A., Kühn, V., and Weber, T. (2008). Iterative Detection for Spatial Multiplexing with Adaptive Power Allocation. In 7th International Conference on Source and Channel Coding (SCC), Ulm.

Ahrens, A. and Lange, C. (2007). Transmit Power Allocation in SVD Equalized Multicarrier Systems. International Journal of Electronics and Communications (AË̈), 61(1):51-61.

Ahrens, A. and Lange, C. (2008). Modulation-Mode and Power Assignment in SVD-equalized MIMO Systems. Facta Universitatis (Series Electronics and Energetics), 21(2):167-181.

Ahrens, A. and Lange, C. (2009). Modulation-Mode and Power Assignment in SVD-assisted MIMO Systems with limited Feedback. In First Asian Conference on e-Business and Telecommunications (CeBT), pages 118, Changhua City (Taiwan).

Bahai, A. R. S. and Saltzberg, B. R. (1999). Multi-Carrier Digital Communications - Theory and Applications of OFDM. Kluwer Academic/Plenum Publishers, New York, Boston, Dordrecht, London, Moskau.

Bingham, J. A. C. (2000). ADSL, VDSL, and Multicarrier Modulation. Wiley, New York.

Choi, R. L. and Murch, R. D. (2003). New Transmit Schemes and Simplified Receivers for MIMO Wireless Communication Systems. IEEE Transactions on Wireless Communications, 2(6):1217-1230.

Choi, R. L. and Murch, R. D. (2004). A Transmit Preprocessing Technique for Multiuser MIMO Systems using a Decomposition Approach. IEEE Transactions on Wireless Communications, 3(1):20-24.

Hanzo, L. and Keller, T. (2006). OFDM and MC-CDMA. Wiley, New York.

Hwang, T. J., Hwang, H. S., and Balik, H. K. (2003). Adaptive OFDM with Channel Predictor over FrequencySelective and Rapid Fading Channel. In Personal, Indoor and Mobile Radio Communications (PIMRC), pages 859-863, Bejing (China).

Jang, J. and Lee, K. B. (2003). Transmit Power Adaptation for Multiuser OFDM Systems. IEEE Journal on Selected Areas in Communications, 21(2):171-178.
Joham, M., Utschick, W., and Nossek, J. A. (2005). Linear Transmit Processing in MIMO Communications Systems. IEEE Transactions on Signal Processing, 53(8):2700-2712.

Krongold, B. S., Ramchandran, K., and Jones, D. L. (2000). Computationally Efficient Optimal Power Allocation Algorithms for Multicarrier Communications Systems. IEEE Transactions on Communications, 48(1):23-27.

Kühn, V. (2006). Wireless Communications over MIMO Channels - Applications to CDMA and Multiple Antenna Systems. Wiley, Chichester.

Liu, W., Yang, L. L., and Hanzo, L. (2008). SVD Assisted Joint Transmitter and Receiver Design for the Downlink of MIMO Systems. In IEEE 68th Vehicular Technology Conference (VTC), pages 1-5, Calgary.

McKay, M. R. and Collings, I. B. (2005). Capacity and Performance of MIMO-BICM with Zero-Forcing Receivers. IEEE Transactions on Communications, 53(1):74- 83.

Meurer, M., Baier, P. W., Weber, T., Lu, Y., and Papathanassiou, A. (2000). Joint Transmission: An Advantageous Downlink Concept for CDMA Mobile Radio Systems using Time Division Duplexing. Electronics Letters, 36(10):900-901.

Mueller-Weinfurtner, S. H. (2002). Coding Approaches for Multiple Antenna Transmission in Fast Fading and OFDM. IEEE Transactions on Signal Processing, 50(10):2442-2450.

Park, C. S. and Lee, K. B. (2004). Transmit Power Allocation for BER Performance Improvement in Multicarrier Systems. IEEE Transactions on Communications, 52(10):1658-1663.

Pätzold, M. (2002). Mobile Fading Channels. Wiley, Chichester.

Proakis, J. G. (2000). Digital Communications. McGrawHill, Boston.

Raleigh, G. G. and Cioffi, J. M. (1998). Spatio-Temporal Coding for Wireless Communication. IEEE Transactions on Communications, 46(3):357-366.

Raleigh, G. G. and Jones, V. K. (1999). Multivariate Modulation and Coding for Wireless Communication. IEEE Journal on Selected Areas in Communications, 17(5):851-866.

van Nee, R. and Prasad, R. (2000). OFDM for Wireless Multimedia Communications. Artech House, Boston and London.

Wong, C. Y., Cheng, R. S., Letaief, K. B., and Murch, R. D. (1999). Multiuser OFDM with Adaptive Subcarrier, Bit, and Power Allocation. IEEE Journal on Selected Areas in Communications, 17(10):1747-1758.

Zheng, L. and Tse, D. N. T. (2003). Diversity and Multiplexing: A Fundamental Tradeoff in MultipleAntenna Channels. IEEE Transactions on Information Theory, 49(5):1073-1096. 


\section{AUTHOR INDEX}

Ahrens, A. .................. 77

Akram, M. .................... 103

Ali, M. ...................44

Aust, S. . . . .................. 77

Awuor, F. ..................... 5

Benavente-Peces, C. . . . . . . . . . . . 77

Bondavalli, A. ................ 115

Chao, H. ......................95

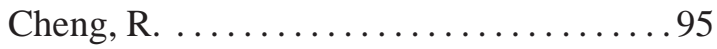

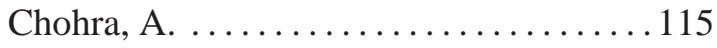

Cuiñas, I. ........................ 35

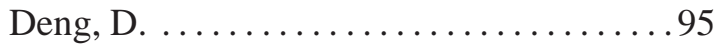

Deng, X. ....................61

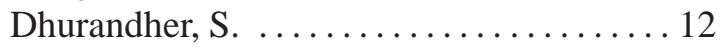

Djouani, K. ................. 5

Dorronzoro, E. ............... 47

Gay-Fernández, J. ................ 35

Giandomenico, F. ................. 115

Gimenes, C. ...................87

Gómez, J. . . ..................47

Guo, J. ..................661

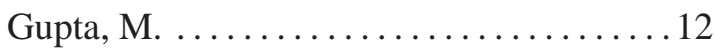

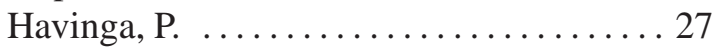

Hong, Y. . . ................... 65

Kleunen, W. ....................27

Lamberti, R. .................. 87

Lee, C. ...................665

Lim, H. . . . . . . . . . . . . . . . 65

Lu, J. . . . ................. 123

Masood, K. .................... 103

Medina, A. ..................47

Meratnia, N. ................. 27

Merino, M. .................44 47

Miridakis, N. . . . . . . . . . . . . . . . . . . 109

Muller, M. ..................... 87

Noel, G. ...................... 5

Obaidat, M. ...............12, 41

Obaidat, M. .................44

Pardiñas-Mir, J. . . . . . . . . . . . . . 87

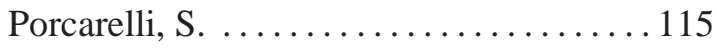

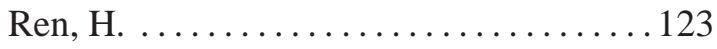

Rivera, O....................47

Selvarajah, K. ................ 21

Shahwan, I. ..................41

Sheikh, A. ................. 103
Speirs, N. ..................21

Stelte, B. .................... . 53

Sultan, F. ... . . . . . . . . . . . . . . . . . 69

Vergados, D. ....................... 109

Wen, H. ....................6 61

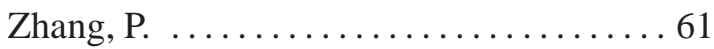

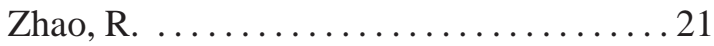

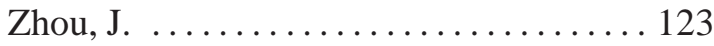

Zummo, S. .................69 